\title{
MỘT CÁCH TIẾP CẬN MỚI TRONG VIỆC GIẢI QUYÊT BÄI TOÁN BIÊN TẬP THỬA ĐẤT SỬ DỤ̂GG CẤU TRÚC DỬ LIỆU DANH SÁCH CẠNH LIÊN KẾT KÉP
}

\author{
ThS. PHAM THÉ HUYNH \\ Trường Đại học Mỏ Địa chất
}

\section{Tóm tắt:}

Hiện nay, khi biên tập mới hoặc chỉnh lý bản đồ địa chính bằng các phần mềm chuyên dụng thì mối quan hệ topo của các thửa đất trên bản đồ không được quan tâm lưu trữ. Mỗi khi có sự thay đổi về thửa đất như thêm đỉnh, bót đỉnh, gộp thửa, tách thửa thì quan hệ topo giữa các thửa bị phá vỡ đồng thời mất đỉ sự liên kết giữa thông tin không gian và thông tin thuộc tính đã được xây dựng. Bài báo này nghiên cứu xây dựng các chức năng biên tập chuyên dụng, đơn giản mà vẫn duy trì được mối quan hệ topo của các thửa đất. Các thuật toán và giải pháp được tác giả xây dựng là cơ sở để hoàn thiện quy trình thành lập bản đồ địa chính trong giai đoạn hiện nay ở Việt Nam.

\section{Mở đầu}

Thuật toán tạo mô hình topo đã được nghiên cứu trong bài báo (Trần Thùy Dương (2006)). Các thuật toán tạo mô hình topo đã được ứng dụng trong các mô đun phần mềm như Picklot, Famis, TMV-Map, AcadMap, ArcTopo ...

Mô hình dữ liệu danh sách cạnh liên kết kép DCEL (Doubly Connected Edge List) được trình bày chi tiết trong (Mark de Berg và ctv (2000)), các mô hình topo khác được mô tả trong các tài liệu (Michael F. Worboys (1995)) và (Peter F. Dale và John D. McLaughlin (1988)). Để thể hiện mối quan hệ topo của các thửa đất hiện nay, chuẩn dữ liệu địa chính (Bộ Tài nguyên và Môi trường (2010)-điều 9) đã quy định chuẩn định dạng dữ liệu và siêu dữ liệu theo ngôn ngư định dạng $\mathrm{GML}, \mathrm{XML}$ với cơ sở là mô hình dữ liệu DCEL.

Tuy nhiên, với các phần mềm biên tập bản đồ địa chính ở Việt Nam hiện nay chỉ tập trung tạo mô hình topo mà chưa chú trọng đến việc lưu trữ mối quan hệ topo của các vùng. Chính vì vậy, mỗi khi có sự biến động về thông tin không gian của vùng cần phải xây dựng lại mô hình topo vừa tốn thời gian, vừa mất đi các thông tin thuộc tính của các vùng đã có. Lúc này, người sử dụng phải gắn thêm các dữ liệu thuộc tính cho các vùng phát sinh. Việc gắn thêm các dữ liệu thuộc tính thường khó kiểm soát bởi các vùng biến động có thể ở nhiều nở trên bản vẽ. Chính vì vậy, người sử dụng thông thường gán lại toàn bộ cơ sở dữ liệu thuộc tính trên bản vẽ khi có bất kỳ một biến động nào về vùng dù chỉ những thay đổi rất nhỏ. Đây là một trong những công việc rất tốn thời gian và dễ nhầm lẫn, bỏ sót trong công tác nội nghiệp.

Để giải quyết vấn đề này, cần phải xây dựng một hệ thống biên tập chuyên dụng với một mô hình cấu trúc dũ liệu topo phù hợp đồng thời có các giải pháp để quản lý cơ sở dữ liệu khi có biến động về vùng mà vẫn đảm bảo mối quan hệ topo giữa các vùng.

\section{Mô tả cấu trúc dữ liệu danh sách cạnh liên kết kép}

Gồm có 3 bảng: Bảng danh sách đỉnh; bảng danh sách nửa cạnh và bảng danh sách vùng. Một cạnh gồm hai nửa cạnh có

Người phản biện: ThS. Nguyễn Phi Sơn 
hướng ngược nhau và nửa cạnh này là nửa cạnh đảo của nửa cạnh kia.

* Bảng danh sách đỉnh: Số hiệu điểm (số nguyên), tọa độ $X$ (số thực), tọa độ $Y$ (số thực)

* Bảng danh sách nửa cạnh: Số hiệu nửa cạnh (số nguyên), số hiệu đỉnh đầu (số nguyên), số hiệu đỉnh cuối (số nguyên), số hiệu vùng bên phải của nửa cạnh (số nguyên), cờ báo hiệu đưa nó vào lịch sử để lưu trữ (lô gíc), nửa cạnh trước (số nguyên), nửa cạnh sau (số nguyên)

* Bảng danh sách vùng: Số hiệu vùng (số nguyên), số hiệu nửa cạnh đường bao, danh sách số hiệu nửa cạnh của các vùng đảo (vùng trong vùng) tương ứng và cờ báo hiệu đưa vùng vào lịch sử để lưu trữ (lô gíc).

Vấn đề đặt ra là làm thế nào để khi biên tập thửa đất (vùng) phải phát sinh dữ liệu để bảo toàn cấu trúc như trên.

\section{Chọn Vùng chỉnh sửa}

Khi tách vùng liên quan đến 2 nửa cạnh $e_{i}, e_{j}$ trong vùng với các điểm phân tách $m$, $\mathrm{n}$ lần lượt thuộc $\mathrm{e}_{\mathrm{i}}, \mathrm{e}_{\mathrm{j}}$. Nối các điểm $\mathrm{m}, \mathrm{n}$ bằng một đường nối (có thể là đoạn thẳng, đường cong hoặc kết hợp nhiều đoạn thẳng, đường cong) sẽ phát sinh ra các điểm mới, các nửa cạnh mới và các vùng mới được đánh số hiệu vùng tăng thêm từ tổng số vùng đã có, đồng thời đưa 2 nửa cạnh $e_{i}, e_{j}$ và vùng bên phải của nửa cạnh $\mathrm{e}_{\mathrm{i}}$ vào lịch sử để lưu trữ.

Khi gộp hai hay nhiều vùng sẽ liên quan đến các cạnh chung của các vùng, lúc này phát sinh một vùng mới là vùng gộp của các vùng có số hiệu vùng là số vùng đã có cộng thêm một.

Quá trình cập nhật bản đồ địa chính với nhiều đơn vị cập nhật khác nhau vào bản đồ tổng thể lưu trữ trong hệ thống quản lý. Vậy cần xử lý như thế nào để không bị xung đột và có thể cập nhật được các dữ liệu đã được biên tập lên toàn hệ thống.

Giả sử chúng ta có một tập hợp các thửa đất liên thông được lưu trữ theo cấu trúc dữ liệu Danh sách cạnh liên kết kép, được tổ chức thành ba cơ sở dữ liệu đỉnh $V_{1}$, cạnh $E_{1}$ và vùng $A_{1}$. Tập hợp các thửa đất liên thông này được gọi là Kết cấu địa chính.

Để biên tập ta cần chọn một tập hợp các thửa đất liên thông thuộc Kết cấu địa chính đưa vào chế độ chỉnh sửa và gọi là Vùng chỉnh sửa. Đường bao của Vùng chỉnh sửa cần được xác định từ danh sách số hiệu thửa đã chọn. Đường bao này có vai trò quan trọng trong việc đánh dấu các thửa đất không được chọn giáp với Vùng chỉnh sửa. Dữ liệu của các thửa đất này và Vùng chỉnh sửa bị cấm chỉnh sửa cho tới khi Vùng chỉnh sửa được chỉnh sửa xong và trả về Kết cấu địa chính để tránh bị xung đột trong quá trình cập nhật biến động.

Các nửa cạnh thuộc đường bao Vùng chỉnh sửa có một tính chất quan trọng là vùng thuộc tính các nửa cạnh đảo của chúng sẽ không nằm trong danh sách số hiệu thửa đất nói trên.

\section{Biên tập thửa đất}

\subsection{Tách thửa}

Ta ký hiệu các đỉnh là v kèm chỉ số, ví dụ như $v_{i}$; các nửa cạnh có kí hiệu là e, ví dụ như $e_{j}$, các vùng kí hiệu là $a$, ví dụ như $a_{k}$. Một thửa đất $a_{i}$ sẽ bao gồm một danh sách các nửa cạnh có thuộc tính vùng là $\mathrm{a}_{\mathrm{i}}$. Các nửa cạnh đảo của các nửa cạnh này sẽ có các thuộc tính vùng là các vùng giáp với $\mathrm{a}_{\mathrm{i}}$.

Khi chỉnh sửa thửa đất bằng các phép dựng hình sẽ phát sinh các đỉnh và các nửa cạnh mới bên trong. Thửa đất này sẽ được chia nhỏ thành các thửa đất mới nằm bên trong. Các thửa đất mới sẽ lát kín thửa đất này.

Trước tiên ta xét việc chia thửa đất làm 
hai bằng một đường chia. Đường chia thửa này có thể có các đầu mút hoặc là nằm trên cạnh hoặc là đỉnh của thửa đẩt được chia.

4.1.1. Hai đầu mút đường chia đều nằm trên cạnh thửa

Giả sử thửa $\mathrm{a}_{2}$ bị chia thành hai thửa mới bằng một đường chia có hai đầu mút nằm trên nửa cạnh $e_{i}$ và $e_{j}$. Hình 1 mô tả các các cạnh $\left(e_{i}, e_{i}^{\prime}\right),\left(e_{j}, e_{j}^{\prime}\right)$ và các vùng $a_{1}, a_{2}, a_{3}$ trước khi vùng $a_{2}$ bị chia. Khi đó $e_{i}$ và $e_{j}$ có cùng thuộc tính vùng là $a_{2}$ còn các nửa cạnh đảo $e_{i}^{\prime}$ và $e_{j}^{\prime}$ sẽ có các thuộc tính vùng tương ứng là $a_{1}$ và $a_{3}$. (Xem hình 1$)$

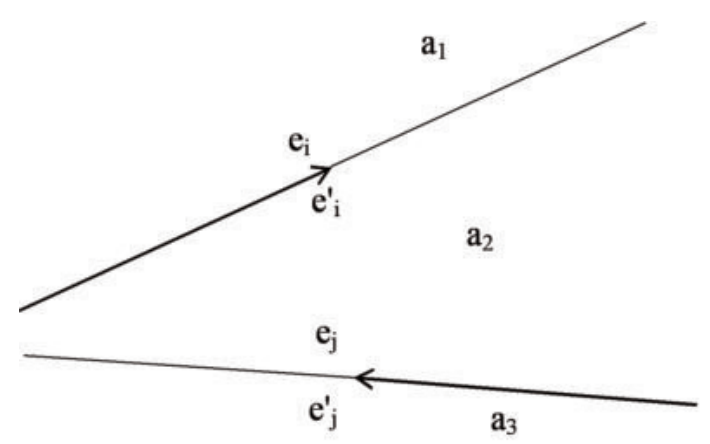

Hình 1: Các cạnh thửa đất trước khi chia

Tại 2 điểm chia trên 2 nửa cạnh $\mathrm{e}_{\mathrm{i}}, \mathrm{e}_{\mathrm{j}}, 2$ nửa cạnh $e_{i}, e_{j}$ và vùng $a_{2}$ sẽ được lưu thành lịch sử, đồng thời phát sinh thêm các nửa cạnh $\mathrm{e}_{\mathrm{i} 1}, \mathrm{e}_{\mathrm{j} 1}, \mathrm{e}_{\mathrm{i} 2}, \mathrm{e}_{\mathrm{j} 2}$. Các nửa cạnh đảo $e_{i}^{i}, e_{j}^{i}$ cũng được xử lý tương tự (Hình 2). Sẽ phát sinh các nửa cạnh $e_{k}, e^{\prime}{ }_{k}$ và các vùng $\mathrm{a}_{4}, \mathrm{a}_{5}$. Các thuộc tính của $\mathrm{e}_{\mathrm{i} 1}, \mathrm{e}_{\mathrm{i} 2}$ sẽ lấy theo thuộc của nửa cạnh $\mathrm{e}_{j}$; các thuộc tính của $e^{\prime}{ }_{11}, e^{\prime}{ }_{i 2}$ lấy theo thuộc tính của nửa cạnh đảo $e_{i}^{\prime}$; tương tự, các thuộc tính của $e_{j 1}, e_{j 2}$ lấy theo nửa cạnh $e_{j}$; còn các thuộc tính của $e_{j 1}^{\prime}, e_{j 2}^{\prime}$ lấy theo nửa cạnh $e_{j}^{\prime}$. Lúc này cơ sở dữ liệu sẽ được lưu trữ với các đỉnh, các nửa cạnh mới, đồng thời phát sinh thêm các vùng liên quan. Việc xét đường chia bằng một cạnh $\left(\mathrm{e}_{\mathrm{k}} \mathrm{e}_{\mathrm{k}}{ }^{\prime}\right)$ làm đơn giản hóa việc phân tích mà không mất tính tổng quát.

Khi xuất hiện điểm chia, cơ sở dữ liệu điểm sẽ được bổ sung. Trong trường hợp này hai điểm chia $v_{1}, v_{2}$ sẽ được thêm vào cơ sở dữ liệu điểm.

\section{Tao vùng $a_{4}$ :}

- xuất phát từ nửa cạnh chia $\mathrm{e}_{\mathrm{k}}$ có điểm đầu là $v_{1}$, điểm cuối là $v_{2}$, xác định $\mathrm{e}_{\mathrm{j} 2}$ có gốc là điểm cuối $v_{2}$ rồi tìm nửa cạnh tiếp theo của $\mathrm{e}_{\mathrm{j} 2}$. Tìm nửa cạnh tiếp theo cho đến khi nửa cạnh tiếp theo là $\mathrm{e}_{\mathrm{i} 1}$ có điểm cuối là $\mathrm{v}_{1}$

- cập nhật thuộc tính cho tất cả nửa cạnh trên vùng thành $a_{4}$

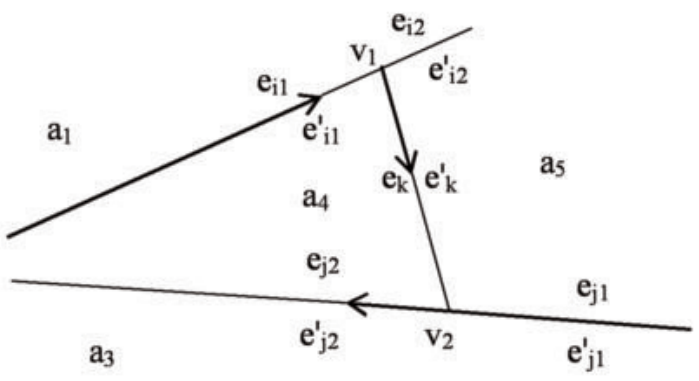

Hình 2: Thửa đất được chia bằng đường chia $e_{k}$ với điểm đầu $v_{1}$ nằm trên cạnh $e_{i}$ và điểm cuối $v_{2}$ nằm trên cạnh $e_{j}$

\section{Tao vùng $a_{\underline{5}}=$}

- Làm tương tự như trên cho nửa cạnh đảo $e^{\prime}{ }_{k}$

4.1.2. Hai đầu mút của đường chia là đỉnh thửa. (Xem hinh 3)

Nếu đầu mút của đường chia là đỉnh sẽ các nửa cạnh mới vả đỉnh mới được tạo ra chỉ nằm trên đường chia $e_{\mathrm{k}}$. Giả sử đường chia xuất phát tại đỉnh $v_{1}$ là điểm gốc của nửa cạnh $\mathrm{e}_{i+1}$ và là điểm cuối của nửa cạnh $\mathrm{e}_{i}$; đường chia $\mathrm{e}_{\mathrm{k}}$ có điểm cuối tại đỉnh $\mathrm{v}_{2}$ là 
điểm gốc của nửa cạnh $\mathrm{e}_{\mathrm{j}+1}$ và là điểm cuối của nửa cạnh $\mathrm{e}_{\mathrm{j}}$

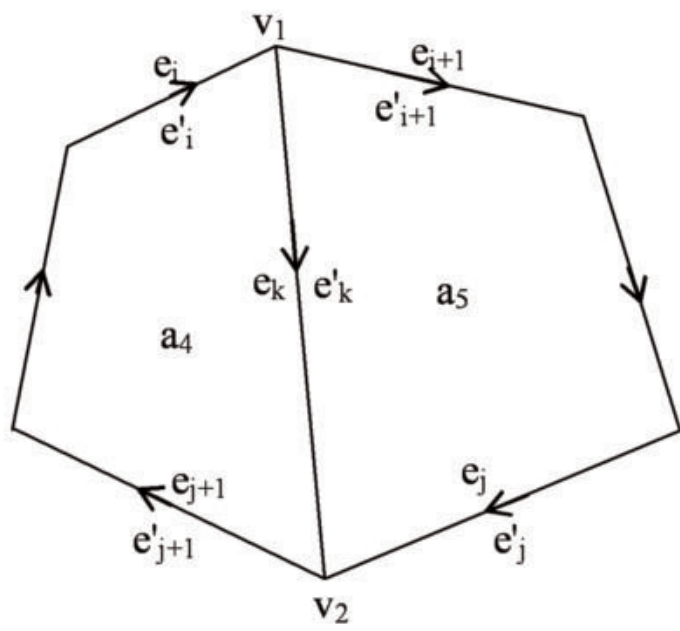

Hình 3: Hai đầu mút đường chia là đỉnh thửa

Quá trình tạo các vùng $\mathrm{a}_{4}$ và $\mathrm{a}_{5}$ được tiến hành tương tự như mô tả ở mục 4.1.1, với vai trò của $e_{i}$ giống như $e_{i 1}, e_{j}$ giống như $e_{j 2}$ ; $e_{i+1}$ giống như $e_{i 2}, e_{j+1}$ giống như $e_{j 1}$

4.1.3. Một đầu mút nằm trên cạnh, một đầu mút là đỉnh thửa

Đối với trường hợp này khi đầu mút của đường chia nằm trên cạnh ta sử dụng cách chia được mô tả ở 4.1.1, còn đối với đầu mút của đường chia là đỉnh thửa ta sử dụng cách chia được mô tả ở 4.1.2.

\subsection{Gộp thửa}

Thao tác gộp thửa trong Vùng chỉnh sửa cần phải xác định đường bao của thửa đất gộp. Duyệt theo các nửa cạnh của từng thửa đất được chọn, nếu vùng thuộc tính các nửa cạnh đảo của nó không nằm trong danh sách số hiệu thửa đất bị gộp thì nửa cạnh này thuộc đường bao thửa đất mới, thuộc tính vùng của nửa cạnh này sẽ được cập nhật; còn nếu ngược lại thì loại cả hai nửa cạnh này và đưa chúng vào lịch sử lưu trữ.

Trong hình 4 , là ví dụ gộp 3 thửa đất $\mathrm{a}_{2}$, $a_{3}, a_{4}$ thành thửa đất $a_{5}$. Các nửa cạnh nét đứt sẽ bị loại bỏ do vùng thuộc tính các nửa cạnh đảo của nó nằm trong danh sách số hiệu thửa đất bị gộp, ngược lại các nửa cạnh nét liền hình thành đường bao thửa đất mới $\mathrm{a}_{5}$. (Xem hình 4)

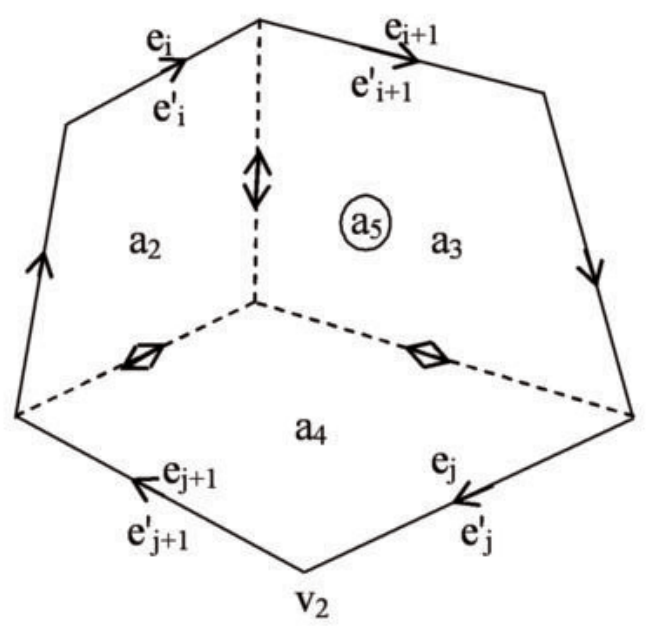

Hình 4: Gộp thửa

\subsection{Thêm bót đỉnh}

Trường hợp tổng quát khi cạnh (gồm hai nửa cạnh $\mathrm{e}_{\mathrm{k}}, \mathrm{e}_{\mathrm{k}}^{\prime}$ đảo nhau) được thay thế bằng một đường đa tuyến có cùng chung điểm đầu và điểm cuối hoặc ngược lại. Ta nhận thấy các cạnh của đường đa tuyến có thuộc tính giống với $\left(e_{k}, e_{k}^{\prime}\right)$, do đó ta chỉ cần sao chép thuộc tính của cạnh $\left(\mathrm{e}_{\mathrm{k}}, \mathrm{e}_{\mathrm{k}}\right)$ cho từng cạnh của đa tuyến hoặc ngược lại và bổ sung hoặc loại bỏ các điểm, các nửa cạnh cần thiết.

\section{Trộn Vùng chỉnh sửa vào Kết cấu địa chính}

Sau khi chỉnh sửa chỉ các yếu tố phát sinh mới được cập nhật vào các cơ sở dữ liệu đỉnh $V_{1}$, nửa cạnh $E_{1}$ và vùng $A_{1}$ tương ưng.

Lúc này Vùng chỉnh sửa sẽ xóa bỏ chế độ cấm chọn để chỉnh sửa và Vùng chỉnh sửa đã được trộn vào Kết cấu địa chính. 


\section{Nghiên cúu}

\section{Kết luận}

Bằng cách cập nhật mô hình dữ liệu DCEL như trình bày ở trên, việc biên tập thửa đất trong Vùng chỉnh sửa có thể được thực hiện dễ dàng mà không làm phá vỡ mô hình cấu trúc dữ liệu topo. $O$

\section{TÀI LIẸU THAM KHẢO}

[1]. Trần Thùy Dương (2006), "Một giải pháp xử lý trường hợp biên trong bài toán tạo Topology", Tạp chí KHKT Mỏ-Địa chất, tập (14), trang 88-91.

[2]. Mark de Berg, Marc van Kreveld, Mark Overmars, Otfried Schwarzkopt, (2000), Computational Geometry, Algorithms and Applications, Springer-
Verlag, Berlin.

[3]. Christine Leslie and Chris Buscaglia (2008), Cadastral Editor Tutorial, ESRI, 380 New York Street, Redlands, CA 923738100, USA.

[4]. Bộ Tài nguyên và Môi trường (2010), Thông tư số 17/2010/TT-BTNMT - Quy định kỹ thuật về chuẩn dữ liệu địa chính

[5]. Michael F. Worboys (1995), GIS : A Computing Perspective, Taylor \& Francis, London.

[6]. Peter F. Dale and John D. McLaughlin (1988), Land Information Management, Clarendon Press, Oxford. $O$

\section{Summary}

New method of parcel editing using data structure of doubly connected edges list MSc. Pham The Huynh

\section{Hanoi University of Mining and Geology}

When editting or modifying cadastral maps by special application nowadays, the maintenance of topology wasn't be interested in. Each time having a change of parcel properties, for example adding and deleting of parcel point, parcel merging or splitting then the topology is broken and the connection bettwen spatial and attribute data is also lost. This article researchs to build the simple special editting functions but keeping this topology. The algorithms and solution introduced by author are basic of improving of cadastral mapping in Vietnam. $\mathrm{O}$

Ngày nhận bài: 13/5/2014. 